\title{
Lactobacillus kefiranofaciens M1 isolated from milk kefir grains ameliorates experimental colitis in vitro and in vivo
}

\author{
Y. P. Chen, ${ }^{*}$ P. J. Hsiao, $†$ W. S. Hong, ${ }^{*}$ T. Y. Dai, ${ }^{*}$ and M. J. Chen ${ }^{*}{ }^{1}$ \\ *Department of Animal Science and Technology, National Taiwan University, 10617 Taipei, Taiwan, ROC \\ †Division of Urology, Cardinal Tien Hospital, 23148 Taipei, Taiwan, ROC \\ ¥Center for Biotechnology, National Taiwan University, 10617 Taipei, Taiwan, ROC
}

\section{ABSTRACT}

Lactobacillus kefiranofaciens M1, isolated from and identified in Taiwanese milk kefir grain, has demonstrated immune-modulating activity. In the present study, we further investigated the effects of $L b$. kefiranofaciens M1 on intestinal epithelial cells in vitro and on dextran sodium sulfate (DSS)-induced colitis in vivo. The possible mechanisms regarding the cytokine products and intestinal epithelial barrier restoration as well as the putative receptor for the protective effects of $L b$. kefiranofaciens M1 were investigated. In vitro results indicated that $L b$. kefiranofaciens M1 could strengthen the epithelial barrier function in vitro by increasing the transepithelial electrical resistance (TEER) and significantly upregulated the level of the chemokine CCL-20 at both the apical and basolateral sites. The in vivo effects of $L b$. kefiranofaciens M1 on the regulation of intestinal physiology indicate that this strain could ameliorate DSS-induced colitis with a significant attenuation of the bleeding score and colon length shortening. Production of proinflammatory cytokines was decreased and that of the antiinflammatory cytokine IL-10 was increased in the DSS-treated mice given $L b$. kefiranofaciens M1. The putative receptor for the protective effects of $L b$. kefiranofaciens M1 was toll-like receptor 2 (TLR2), which was involved in probiotic-induced cytokine production in vitro and in attenuation of the bleeding score and colon length shortening in vivo. In this study, the kefir lactobacillus Lb. kefiranofaciens M1 clearly demonstrated an anticolitis effect. Based on these results, Lb. kefiranofaciens M1 has the potential to be applied in fermented dairy products as an alternative therapy for intestinal disorders.

Key words: kefir, probiotic, intestinal epithelial cell, experimental colitis

Received July 6, 2011.

Accepted September 25, 2011.

${ }^{1}$ Corresponding author: cmj@ntu.edu.tw

\section{INTRODUCTION}

The intestinal epithelial barrier, which prevents luminal microbial content from entering the lamina propria, is important in intestinal homeostasis (Marchiando et al., 2010). Because intestinal barrier function can be regulated by intestinal microbes through various mechanisms (Neish, 2004), a potential therapeutic application has been proposed that uses a probioticbased treatment to regulate intestinal barrier function in intestinal inflammation-related disease (Geier et al., 2007; Vanderpool et al., 2008). In many clinical trials, probiotics have demonstrated ameliorating effects in treating intestinal diseases, including ulcerative colitis, irritable bowel syndrome, Clostridium difficile infection, infectious and antibiotic-associated diarrhea, and necrotizing enterocolitis (Gareau et al., 2010). Various studies have demonstrated that probiotics are able to increase the ability of the intestinal epithelial barrier to inhibit pathogenic bacteria attaching to the gut mucosa (Johnson-Henry et al., 2008; Reiff and Kelly, 2010). Probiotics are also capable of upregulating expression of defensins (Schlee et al., 2008), mucins (CaballeroFranco et al., 2007), and proteins related to tight junctions (Ukena et al., 2007). Additionally, probiotics illustrate the potential to regulate immune responses through regulatory T cells and dendritic cell (DC; Reiff et al., 2009). Pronio et al. (2008) reported that after administration of a probiotic cocktail, VSL\#3, composed of 8 different probiotic strains, patients with ulcerative colitis showed a reduction in the pouchitis disease activity index and an increase in the number of mucosal regulatory T cells. Another study performed by Hoarau et al. (2008) indicated that a fermentation product of Bifidobacterium breve was able to differentially activate mitogen-activated protein kinases (MAPK), phosphatidylinositol 3-kinase (PI3K), and glycogen synthase kinase-3 (GSK3) to regulate the maturation and survival of DC.

Among different probiotic strains, lactobacilli such as Lactobacillus acidophilus (Chen et al., 2009), Lactobacillus paracasei (Mileti et al., 2009), Lactobacillus rhamno- 
sus (Miyauchi et al., 2009), and Lactobacillus crispatus (Castagliuolo et al., 2005; Voltan et al., 2007, 2008) have been widely investigated for potential protective probiotic effects in a dextran sodium sulfate (DSS)induced colitis model. Many possible mechanisms are involved in the lactobacilli-elicited protective effects in DSS-induced colitis in mice, including antiinflammatory effects (Mileti et al., 2009), regulation of intestinal barrier function, increase in barrier regulating proteins (Miyauchi et al., 2009), and activation of peroxisome proliferator-activated receptor gamma (Castagliuolo et al., 2005; Voltan et al., 2007, 2008).

Toll-like receptors (TLR) have been widely investigated because of their importance in microbe recognition. Rakoff-Nahoum et al. (2004) showed that the absence of TLR2, TLR4, or myeloid differentiation primary response gene (88) (MyD88) in mice resulted in severe colitis induced by DSS administration, and that colitis was more severe when commensal bacteria were depleted by antibiotics. In the polarized intestinal epithelial cell models HT-29 and Caco-2, activation of TLR2 could enhance barrier integrity through protein kinase $\mathrm{C}$ activation and ZO-1 distribution in tight junctions (Cario et al., 2004). The authors further proved that oral administration of the TLR2 ligand Pam3csk4 could suppress DSS-induced colitis in mice (Cario et al., 2007). These findings show that TLR2 activation could be a therapeutic method of colitis treatment. However, few studies have investigated the possible role of TLR2 in the underlying mechanisms of the protective effects of probiotics on colitis (Vanderpool et al., 2008; Ng et al., 2009).

Kefir is an alcoholic, fermented milk beverage produced by the fermentation of kefir grains, which contain lactic acid bacteria, acetic acid bacteria, and yeasts (Farnworth and Mainville, 2003). Kefir shows many different health-promoting abilities (Farnworth and Mainville, 2003), such as antitumor (Liu et al., 2002), antimutagenic (Liu et al., 2005), antioxidant (Liu et al., 2005), and hypocholesterolemic effects (Liu et al., 2006). Lactobacillus kefiranofaciens, isolated from kefir, is a heterofermentative bacterium that produces ethanol, acetic acid, carbon dioxide, diacetyl, acetoin, 2-3-butanediol, and formate, in addition to lactic acid. Lactobacillus kefiranofaciens has also been reported as a kefiran (exopolysaccharide) producer in kefir. However, few studies have reported the functional properties of Lb. kefiranofaciens.

Lactobacillus kefiranofaciens M1, previously isolated in our laboratory from Taiwanese milk kefir grain (Chen et al., 2008), demonstrated immune-modulating activity in vitro (Hong et al., 2009). An increase in the production of regulatory T-cell cytokines was observed when Lb kefiranofaciens M1 was cocultured with spleen cells (our unpublished data). Thus, in this study, we investigated the effects of Lb. kefiranofaciens M1 on intestinal epithelial cells in vitro and DSS-induced colitis in vivo. The possible mechanisms regarding the cytokine products and intestinal epithelial barrier restoration as well as the putative receptor for the protective effects of $L b$. kefiranofaciens M1 were investigated.

\section{MATERIAL AND METHODS}

\section{Lactic Acid Bacterial Sample Preparation}

Lactobacillus kefiranofaciens M1 was isolated and identified as previously demonstrated (Chen et al., 2008). The lactobacilli were cultured in de Man, Rogosa, and Sharpe broth (Difco Laboratories, Detroit, MI) at $37^{\circ} \mathrm{C}$ and harvested in log phase (optical density value $=0.8$ ) by washing and resuspending 3 times in PBS (Hyclone, South Logan, UT). After washing, the lactic acid bacteria (LAB) cells were resuspended in PBS and adjusted to desired concentrations.

\section{Caco-2 Epithelial Monolayer}

Human colonic epithelial cell line Caco2-C2BBe1 was obtained from American Type Culture Collection (Manassas, VA) and cultured in Dulbecco's modified Eagle's medium supplemented with $10 \%$ heat-inactivated fetal bovine serum, $50 \mu \mathrm{g} / \mathrm{mL}$ penicillin, $50 \mu \mathrm{g} /$ $\mathrm{mL}$ streptomycin sulfate, and $100 \mu \mathrm{g} / \mathrm{mL}$ neomycin sulfate (Invitrogen, Carlsbad, CA). All cells were cultured in a humidified atmosphere of $5 \% \mathrm{CO}_{2}$ at $37^{\circ} \mathrm{C}$. The intestinal epithelial monolayer was formed by using Caco-2 cells seeded onto permeable 12-well Transwell membranes (Corning, Lowell, MA) with a $3-\mu \mathrm{m}$ pore size (density, $10^{5} / \mathrm{cm}^{2}$ ). The culture medium was replaced with fresh medium every $2 \mathrm{~d}$ during the 28 -d culture period. After $28 \mathrm{~d}$, the transepithelial electrical resistance (TEER) of the Caco-2 epithelial monolayers was greater than $300 \Omega \cdot \mathrm{cm}^{2}$, indicating that the monolayers were ready to use (Klingberg et al., 2005). The TEER was measured using an epithelial volt-ohmmeter with an STX2 probe (World Precision Instruments, Sarasota, FL).

\section{Effect of Lb. kefiranofaciens M1 on Integrity of Caco-2 Epithelial Monolayer}

The Caco- 2 monolayers were cocultured with $10^{6}$ to $10^{8} \mathrm{cfu} / \mathrm{mL}$ of $L b$. kefiranofaciens $\mathrm{M} 1$ at $37^{\circ} \mathrm{C}$ for 24 $\mathrm{h}$, followed by the addition of $3 \%$ DSS for $30 \mathrm{~h}$ (Araki et al., 2006). The TEER values before coculture with M1 and after treatment with DSS were measured. The TEER was measured before the addition of the bacte- 
ria (time zero) and then at various time intervals and expressed as the ratio of the TEER at time $t$ to the initial value (at time zero) for each series. The TEER of monolayers without added bacteria represented the controls for each experiment.

Macromolecule permeability was also evaluated to determine the integrity of Caco-2 epithelial monolayers ( $\mathrm{Li}$ et al., 2003). After treating with LAB, fluorescein isothiocyanate (FITC)-labeled dextran (average molecular weight: 10,000 Da, Sigma, St. Louis, MO) was added to the apical site of the Caco-2 monolayer. Twenty microliters of basolateral medium was collected at different time points and then diluted 10-fold. The quantification of fluorescence in the basolateral medium was measured using a Victor2 fluorescence counter (PerkinElmer, Waltham, MA) at excitation and emission wavelengths of $492 \mathrm{~nm}$ and $515 \mathrm{~nm}$, respectively.

\section{DSS-Induced Colitis Animal Model}

The DSS-induced colitis model in mice was modified from the method of Wirtz et al. (2007). Specific-pathogen-free female C57BL/6 mice 8 to 10 wk old were purchased from National Laboratory Animal Center (Taipei, Taiwan), and TLR2 knockout mice with a B6/129 background were purchased from the Jackson Laboratory (Bar Harbor, ME). The mice were maintained in a standard cage environment at $25^{\circ} \mathrm{C}$ and exposed to a 12-h light and dark cycle. Mice with similar BW were separated into groups of 8 mice and each was administrated PBS, $10^{7}$ cfu of $L b$. kefiranofaciens M1, or $10^{8}$ cfu of Lb. kefiranofaciens M1 intragastrically daily for $14 \mathrm{~d}$. During the last $7 \mathrm{~d}$ of administration, 2\% DSS (molecular weight: 36,000-50,000 Da; MP Biomedicals, Aurora, $\mathrm{OH}$ ) was added to the drinking water to induce colitis. The 2\% DSS solution was replaced with fresh DSS every $2 \mathrm{~d}$. The mice were anesthetized by using isoflurane (Abbott Laboratories, Kent, UK) and then killed by cervical dislocation. The colon was removed and the length of the colon was measured. The feces in the colon were removed by flushing with ice-cold PBS. A 1-cm-long fragment from the distal part of colon was excised, washed with ice-cold PBS, and immediately immersed in 10\% histological-grade phosphate-buffered formalin (Mallinckrodt Chemical, Derbyshire, UK).

\section{Intestinal Bleeding Assessment}

For collection of feces, mice were removed from their cages and placed individually in circular plastic containers $(15 \mathrm{~cm}$ diameter $\times 18 \mathrm{~cm}$ high) with filter paper on the bottom. Fecal pellets were collected (approximately 150-200 mg of feces per sample) after $1 \mathrm{~h}$ of isolation. Collections were made from each of the DSS-treated and untreated mice. The intestinal bleeding score system (Wirtz et al., 2007) assessed stool consistency and bleeding. Occult blood in the feces was measured using Hemoccult Sensa (Beckman Coulter, Brea, CA).

\section{Histological Evaluation}

Fixed colon tissue samples were dehydrated in ethanol and further embedded in paraffin wax, then sectioned (5 $\mathrm{m}$ thickness) and stained with hematoxylin and eosin stain. The histological score was evaluated according to Wirtz et al. (2007) by well-trained histologists.

\section{Colon Organ Culture and Cytokine Production}

Colonic cytokine production was measured by ex vivo colon organ culture (Gibson et al., 2008). A colon segment $(1 \mathrm{~cm})$ was cut longitudinally and washed in icecold PBS with protease inhibitor cocktails (Sigma) and $50 \mu \mathrm{g} / \mathrm{mL}$ penicillin, $50 \mu \mathrm{g} / \mathrm{mL}$ streptomycin sulfate, and $100 \mu \mathrm{g} / \mathrm{mL}$ neomycin sulfate (Invitrogen). After washing, the segment was cultured in 24-well culture plates (BD Biosciences, San Jose, CA) in serum-free RPMI-1640 medium (Invitrogen) supplemented with protease inhibitor cocktails and $50 \mu \mathrm{g} / \mathrm{mL}$ penicillin, 50 $\mu \mathrm{g} / \mathrm{mL}$ streptomycin sulfate, and $100 \mu \mathrm{g} / \mathrm{mL}$ neomycin sulfate for $24 \mathrm{~h}$ in a humidified atmosphere of $5 \% \mathrm{CO}_{2}$ at $37^{\circ} \mathrm{C}$. The supernatants were collected by centrifugation at $13,400 \times g$ and $4^{\circ} \mathrm{C}$ for $3 \mathrm{~min}$ to remove tissue debris and then stored at $-80^{\circ} \mathrm{C}$ until ELISA.

The cytokine levels in the supernatants were measured by DuoSet ELISA development systems (R\&D Systems, Minneapolis, MN) according to the manufacturer's instructions.

\section{Reporter Gene Assay}

The reporter gene assay was modified from the method of Karczewski et al. (2010). Cells from the human embryonic kidney epithelial cell line HEK293T $\left(2.5 \times 10^{4}\right.$ cells/well $)$ were seeded onto 96 -well plates and cultured overnight. The cells were transfected using Lipofectamine 2000 (Invitrogen) plus $0.01 \mu \mathrm{g}$ of TLR2-expressing plasmid pUNO-hTLR2 (Invitrogen), $0.07 \mu \mathrm{g}$ of p5xNFkB-luc plasmid (Stratagene, Cedar Creek, TX), and $0.02 \mu \mathrm{g}$ of pcDNA3.1-3gal for $24 \mathrm{~h}$ according to the manufacturer's instructions. The cells were incubated with either PBS or probiotic sample for $6 \mathrm{~h}$, washed twice with PBS, and then lysed. Nuclear factor $\kappa \mathrm{B}(\mathbf{N F} \kappa \mathbf{B})$ luciferase activities were measured using a luciferase assay system (Promega, Madison, WI) according to the manufacturer's instructions. Levels of firefly luciferase expression were normalized 
against $\beta$-galactosidase activity as a control for transfection efficiency and expressed as fold stimulation over unstimulated pcDNA3.1 empty vector control.

\section{Antibody Blocking Test}

For TLR2 blocking experiments, Caco-2 monolayers were treated with $10 \mu \mathrm{g} / \mathrm{mL}$ mouse monoclonal anti-mouse/human TLR2 antibody for functional assay (eBioscience, San Diego, CA) or $10 \mu \mathrm{g} / \mathrm{mL}$ mouse isotype control IgG (Pierce, Rockford, IL) at the apical site for $1 \mathrm{~h}$ before treatment (Meng et al., 2004). Cytokine production in the culture medium of the apical and basolateral sites was measured $24 \mathrm{~h}$ later by ELISA.

\section{Statistical Analysis}

The values are given as mean \pm standard deviation. All results were analyzed by Duncan's multiple range test with SAS software (SAS Institute Inc., Cary, NC). A $P$-value $<0.05$ indicated a significant difference.

\section{RESULTS}

\section{Lb. kefiranofaciens M1 Enhances Intestinal Epithelial Barrier Function In Vitro}

The in vitro intestinal epithelial barrier function was investigated using the polarized intestinal epithelial monolayer formed by the Caco-2 cell line. Lactobacillus kefiranofaciens M1 increased the TEER of polarized Caco-2 monolayers in a dose-dependent manner, whereas administration of only $10^{8} \mathrm{cfu} / \mathrm{d}$ of M1 showed a significant change $(P<0.05)$ with $25 \%$ enhancement (Figure 1). Chemokines involved in intestinal homeostasis or epithelial restitution were also evaluated (Figure 2). The chemokine CCL-20, which mediates restitution of colonic epithelial cells, was significantly upregulated at both the apical and basolateral sites in the Caco- 2 monolayer treated with $10^{8} \mathrm{cfu} / \mathrm{d}$ of $\mathrm{Lb}$. $\mathrm{ke}$ firanofaciens M1 at the apical site (Figure 2A). In contrast, CXCL-12, an intestinal epithelial restitution and migration chemokine, and IL-8, a neutrophil-attracting chemokine, showed no significant changes at either the apical or basolateral site after Lb. kefiranofaciens M1 treatment (Figure 2B and $\mathrm{C}$ ).

\section{Lb. kefiranofaciens M1 Protects Intestinal Epithelial Monolayer Against DSS Challenge In Vitro}

Because Lb. kefiranofaciens M1 can enhance barrier integrity, we further assessed Lb. kefiranofaciens M1 in protection of the intestinal epithelial monolayer against DSS challenge. Results indicated that the DSS chal- lenge significantly damaged intestinal epithelial cells, with an $80 \%$ reduction of the TEER value within 0 to $3 \mathrm{~h}$ at the apical site (Figure 3A). However, after $22 \mathrm{~h}$, the TEER value of the Lb. kefiranofaciens M1-treated group was significantly increased compared with that of the controls (Figure 3A). In addition, the DSSinduced basolateral influx of FITC-labeled dextran was dramatically increased after 3\% DSS treatment for 18 h (Figure 3B). The treatment of Lb. kefiranofaciens M1 at the apical site significantly decreased the DSSinduced dextran influx (Figure 3B), which indicated that the macromolecule leakage of the Caco- 2 monolayer had improved. The in vitro data show that $L b$. kefiranofaciens M1 can protect the intestinal epithelial barrier against DSS-induced damage.

\section{Lb. kefiranofaciens M1 Ameliorates DSS-Induced Colitis In Vivo}

The anticolitis potential of Lb. kefiranofaciens M1 in vivo was subsequently assessed. Mice administrated $L b$. kefiranofaciens M1 showed a lower rectal bleeding score than the colitis control mice (Figure 4A). This effect became statistically significant in mice fed $10^{8} \mathrm{cfu} / \mathrm{d}$ of $L b$. kefiranofaciens M1. The mice in the DSS colitis group showed a significant reduction in the length of the colon, which was attenuated in the $L b$. kefiranofaciens M1 groups in a dose-dependent manner (Figure 4B). The spleen weights of the $L b$. kefiranofaciens M1-treated groups were lower than those of the colitis controls, but the differences were not statistically significant (Figure 4C).

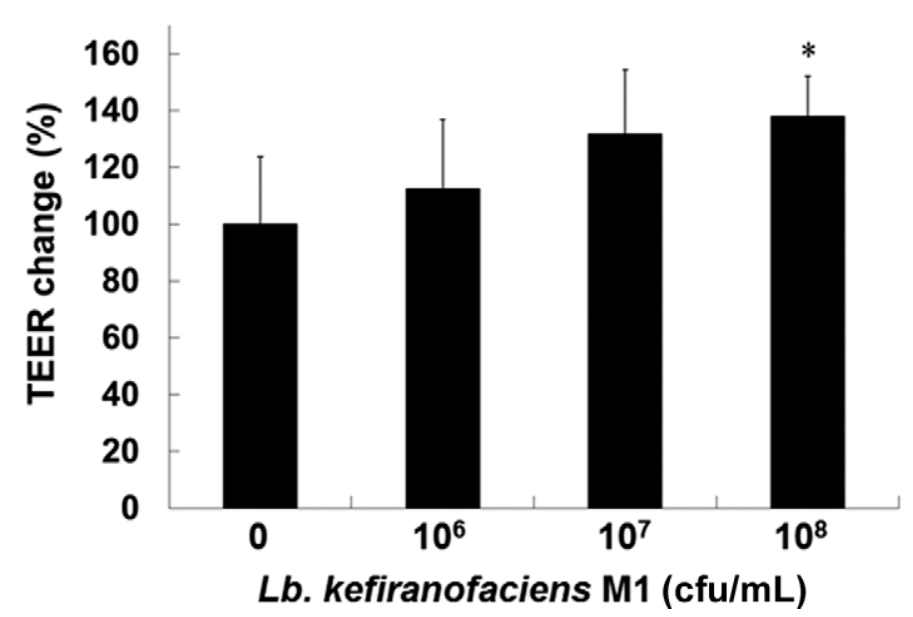

Figure 1. Lactobacillus kefiranofaciens M1 increases transepithelial electric resistance (TEER) of Caco-2 monolayer. The TEER values of Caco-2 monolayers were measured after treating with $L b$. kefiranofaciens $\mathrm{M} 1$ in indicated dosages at the apical site for $24 \mathrm{~h} .{ }^{*} P<0.05$ compared with the nontreated control. 
(A)

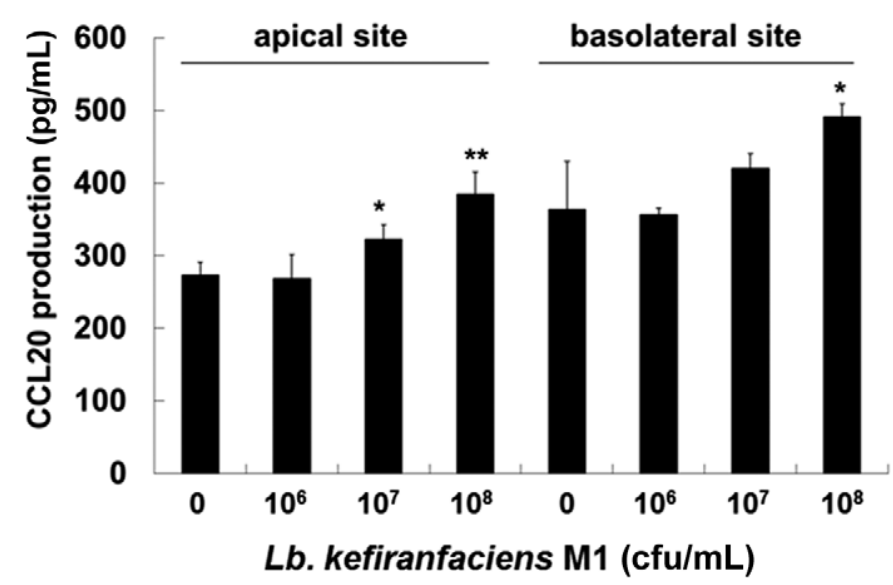

(B)

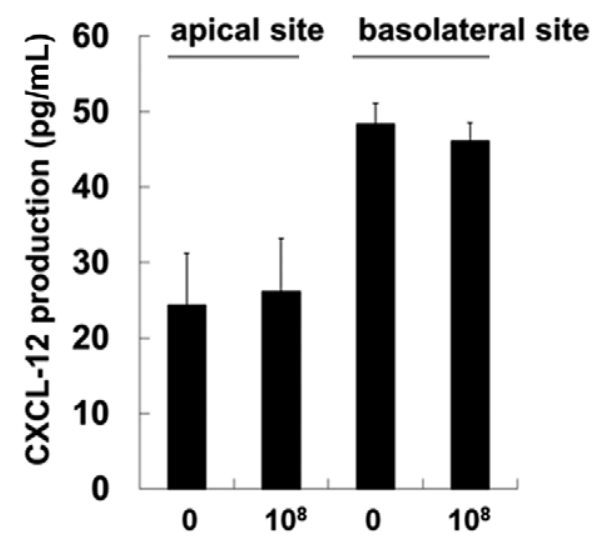

Lb. kefiranfaciens M1 (cfu/mL)

(C)

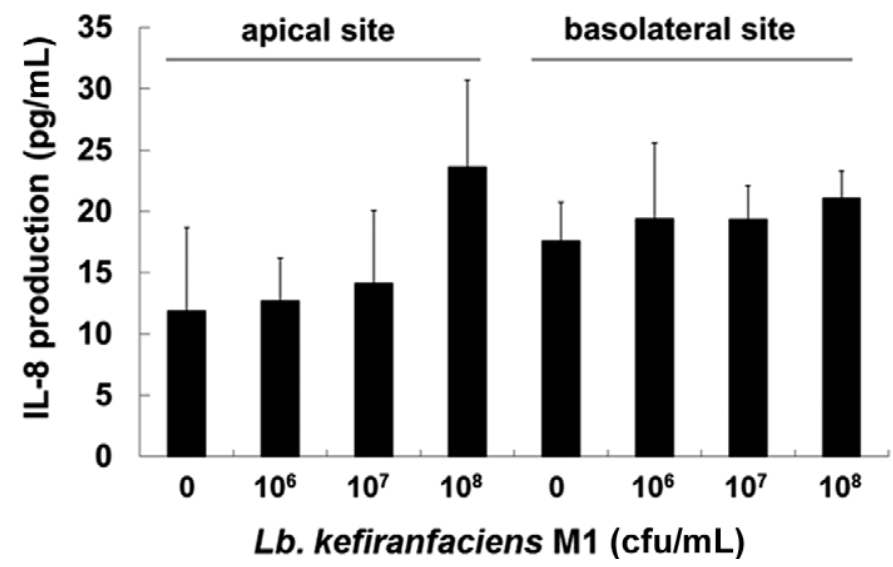

Figure 2. Cytokine production of Caco-2 monolayer stimulated with Lactobacillus kefiranofaciens M1. Caco-2 monolayers were treated with $L b$. kefiranofaciens M1 in indicated dosages at apical site for $24 \mathrm{~h}$. Medium was collected from apical and basolateral sites, respectively, and production of cytokines (A) CCL-20, (B) CXCL-12, and (C) IL-8 was measured by ELISA. ${ }^{*} P<0.05$, ${ }^{* *} P<0.01$ compared with the nontreated control.
(A)

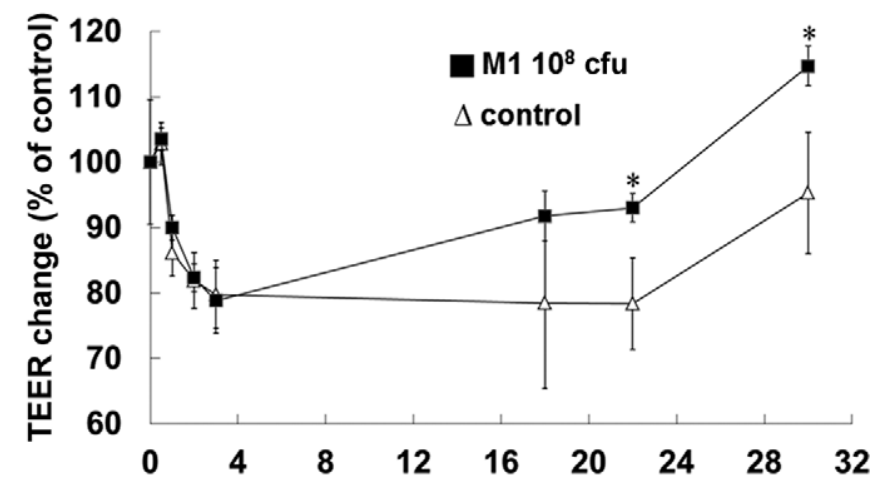

(B)

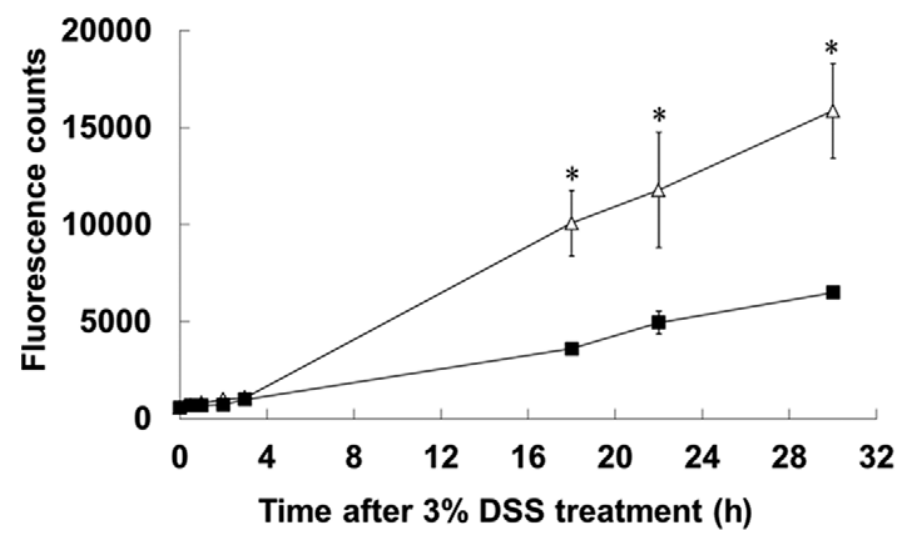

Figure 3. Lactobacillus kefiranofaciens M1 protects barrier function of Caco-2 monolayer against dextran sodium sulfate (DSS)-induced damage. Caco-2 monolayers were pretreated with $10^{8} \mathrm{cfu}$ of $\mathrm{Lb}$. $\mathrm{ke}$ firanofaciens M1 at apical site for $24 \mathrm{~h}$ and washed. Then, DSS 3\% was added at the apical site at different time points. Transepithelial electric resistance (TEER) values (A) and fluorescence counts of fluorescein isothiocyanate-dextran in basolateral site (B) were measured. ${ }^{*} P<0.01$ compared with the nontreated control.

Histological analysis showed that the colitis control animals displayed signs of severe colitis with a high degree of inflammatory infiltrates in the colonic mucosa, loss of goblet cells, and a disturbed mucosal architecture. On the other hand, inflammation was significantly reduced in mice treated with both $10^{7}$ and $10^{8} \mathrm{cfu} / \mathrm{d}$ of Lb. kefiranofaciens M1 (Figure 5A to D), reflected by improvements in the inflammatory scores from 3.2 to 1.8 and from 3.2 to 2.0 , respectively (Figure 5E).

\section{Cytokines Secretion in Colonic Organ Culture}

The cytokine production profile in colonic organ culture ex vivo showed results that support the ameliorating ability of Lb. kefiranofaciens M1. Secretion of 2 proinflammatory cytokines, IL- $1 \beta$ and $\mathrm{TNF} \alpha$, were significantly increased in animals that received DSS (Figure 6). The administration of $10^{7}$ or $10^{8} \mathrm{cfu} / \mathrm{d}$ of 


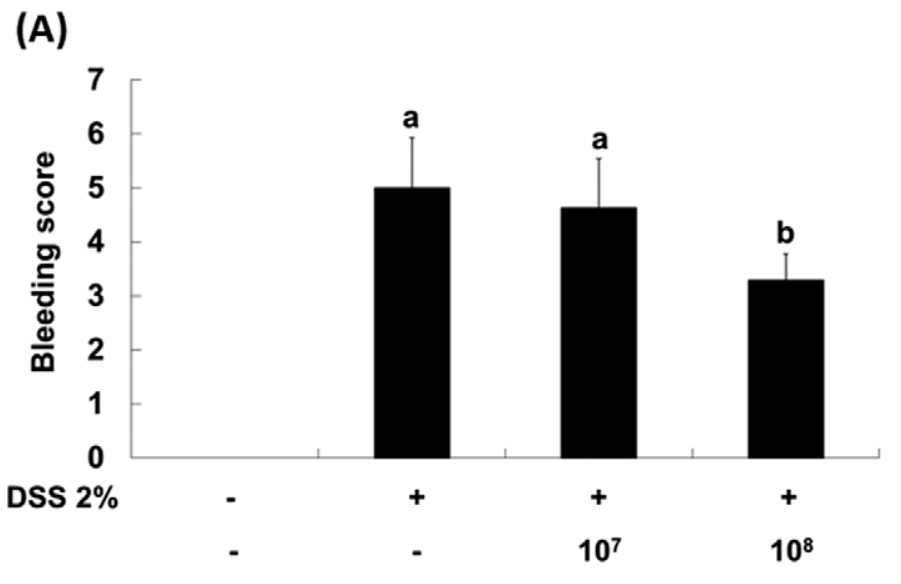

(B)

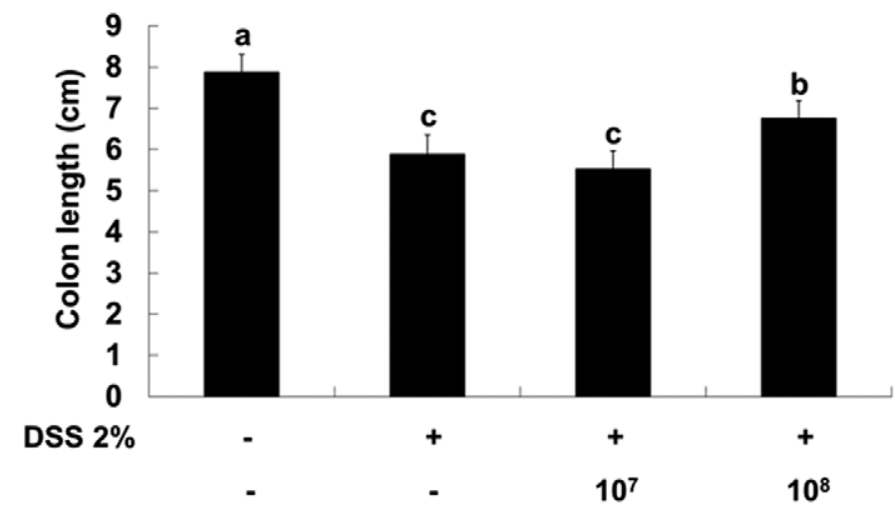

(C)

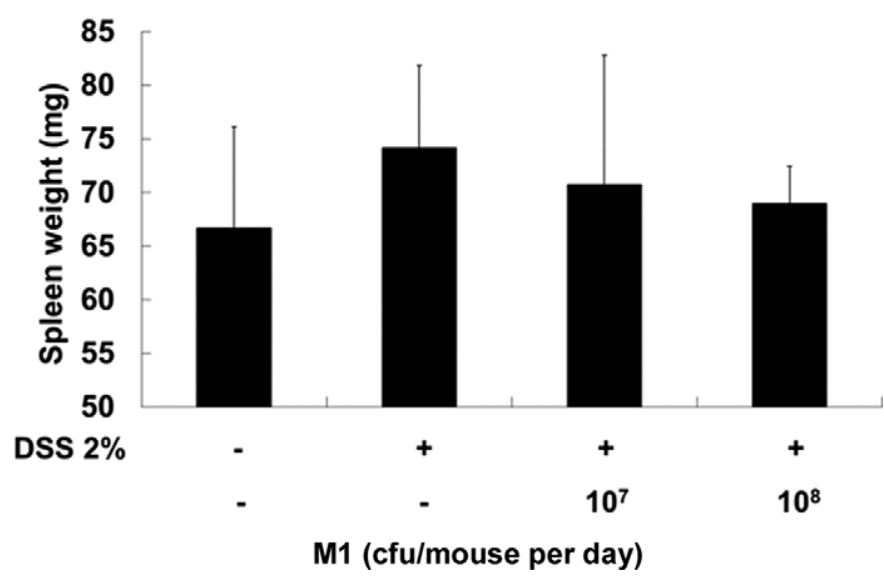

Figure 4. Lactobacillus kefiranofaciens M1 $\left(10^{7}\right.$ or $\left.10^{8} \mathrm{cfu} / \mathrm{d}\right)$ ameliorates dextran sodium sulfate (DSS)-induced colitis in mice. The bleeding score (A) was evaluated on the last day of treatment. After the mice were killed, the colon and spleen were removed and the length of colon (B) and the weight of spleen (C) were measured. Means for data without a common letter differ significantly $(P<0.05)$.

Lb. kefiranofaciens M1 significantly inhibited the production of IL-1 $\beta$ and TNF- $\alpha$ induced by DSS (Figure $6)$. Conversely, the production of the antiinflammatory and regulatory cytokine IL-10 was significantly increased in the Lb. kefiranofaciens M1-treated groups compared with the DSS group (Figure 6).

\section{TLR2 Plays an Important Role Through NFKB in Protective Effects of $L b$. kefiranofaciens M1 on DSS-Induced Colitis}

The putative receptor for the protective effects of $L b$. kefiranofaciens M1 was investigated in vitro and in vivo. In vitro results indicated that $L b$. kefiranofaciens M1 $\left(10^{8} \mathrm{cfu} / \mathrm{mL}\right)$ significantly induced NFKB activation when TLR2 was present in TLR-lacking HEK293T cells, whereas the production of $\mathrm{NF} \kappa \mathrm{B}$ was low for treatment without TLR2 and controls (Figure 7A). Additionally, the CCL20 levels induced by Lb. kefiranofaciens M1 at both the apical and basolateral sites were significantly decreased when the Caco-2 monolayer was pretreated with TLR2-specific antibodies without isotype control IgG (Figure 7B).

In vivo results confirmed the previous in vitro findings. Amelioration of DSS-induced colitis with administration of M1, as shown by a lower bleeding score (Figure 8A) and maintenance of colon length (Figure $8 \mathrm{~B}$ ), was not found in the TLR2 knockout mice. The TLR2 knockout mice showed a significantly higher bleeding score and shorter colon length compared with the wild-type group.

\section{DISCUSSION}

In the present study, we first demonstrated that $L b$. kefiranofaciens M1 could strengthen the epithelial barrier function in vitro by increasing the TEER (Figure 1 ), a sensitive method for determining permeability in vitro (Klingberg et al., 2005). In other words, treatment with Lb. kefiranofaciens M1 might prevent or reverse increased permeability of the epithelium. A similar increase in the TEER of polarized Caco- 2 cell monolayers has been observed by other investigators following the addition of various probiotics in vitro. Madsen et al. (2001) showed that a probiotic compound, VSL\#3, composed of 8 different probiotic strains, could enhance TEER in the T84 intestinal epithelial cell monolayer. Recently, Lb. plantarum MF 1298, Lactobacillus salivarius DC5 (Klingberg et al., 2005), Streptococcus thermophilus, Lb. acidophilus (Resta-Lenert and Barrett, 2003, 2006), Escherichia coli Nissle 1917 (Zyrek et al., 2007), and Lb. plantarum (Anderson et al., 2010; Karczewski et al., 2010) have also been reported to enhance TEER and resist proinflammatory cytokine- or pathogen-induced barrier dysfunction in different intestinal epithelial cell monolayers.

Previous studies have suggested that certain chemokines, such as CCL-20 and CXCL-12, might be involved 
PBS

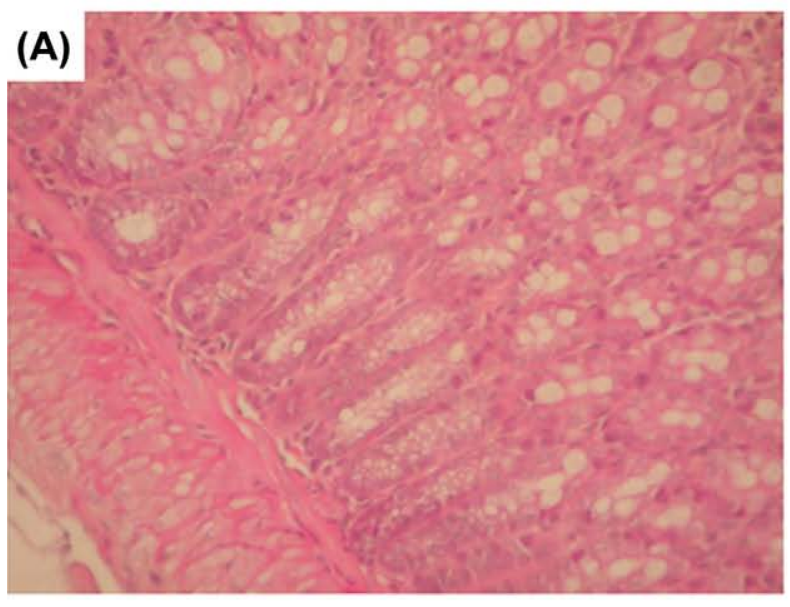

DSS + M1 (107 cfu/mouse per day)

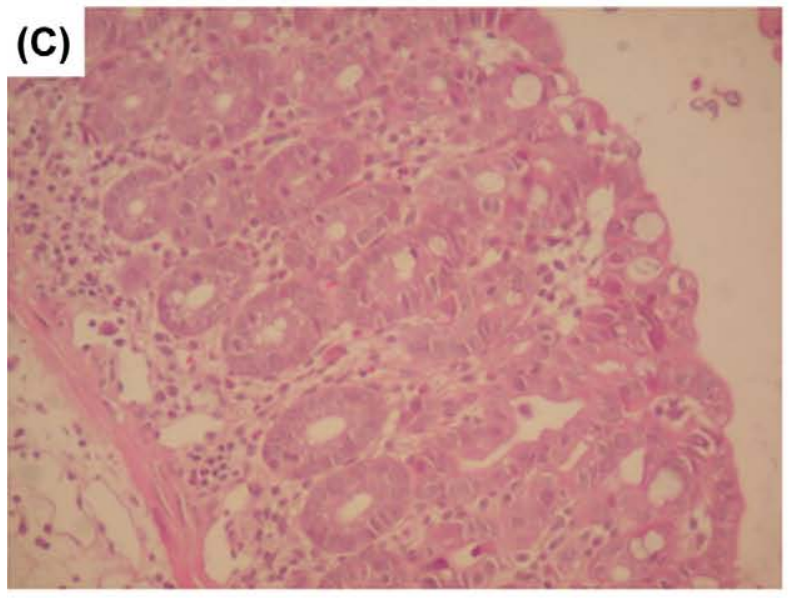

DSS

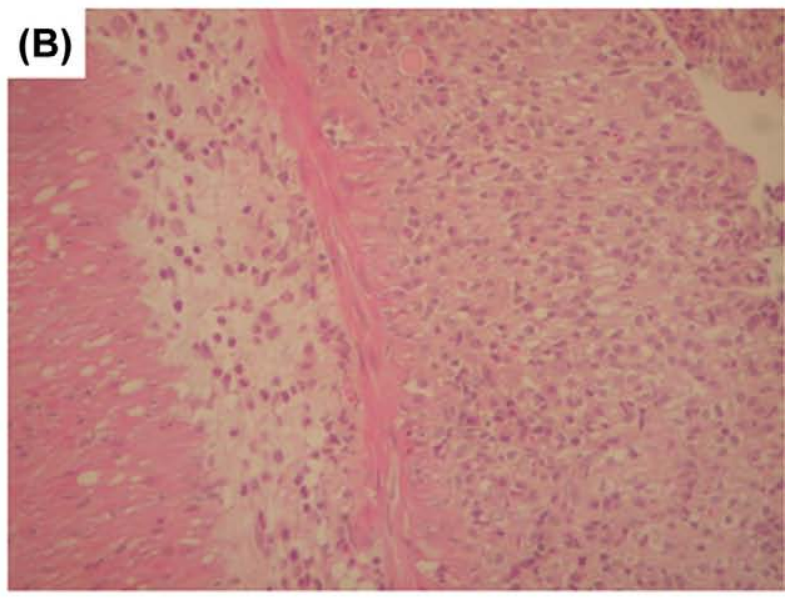

\section{DSS + M1 (10 $10^{8}$ cfu/mouse per day)}

(D)

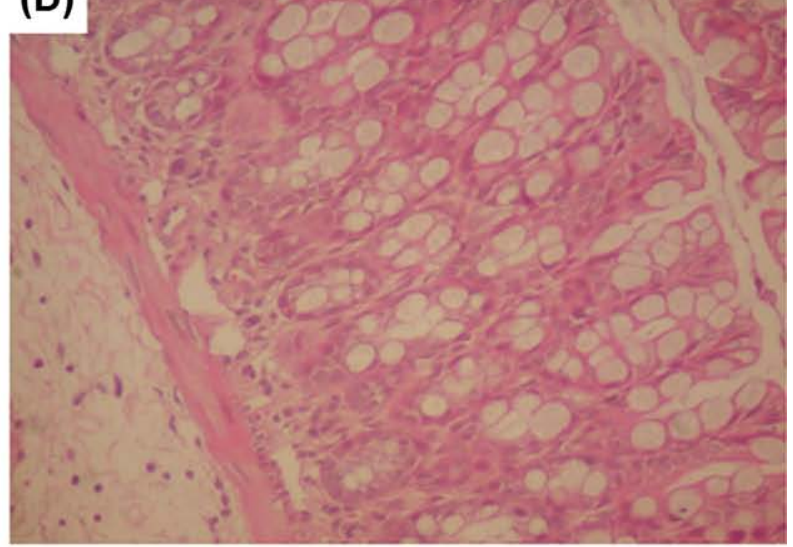

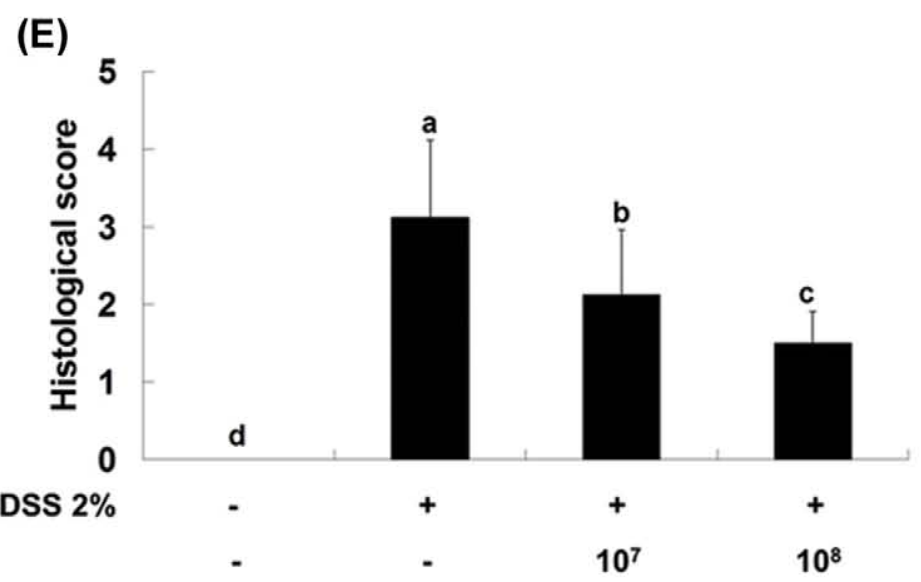

\section{M1 (cfu/mouse per day)}

Figure 5. Representative histological photographs and quantitative histological scores of distal colon section of mice with different treatments in dextran sodium sulfate (DSS)-induced colitis model. At the end of DSS-induced colitis model, mice were killed and distal colons were collected followed by fixation and hematoxylin and eosin staining (A to D). Histological scores were evaluated (E), and means for data without a common letter differ significantly $(P<0.05)$. Color version available in the online PDF. 
(A)

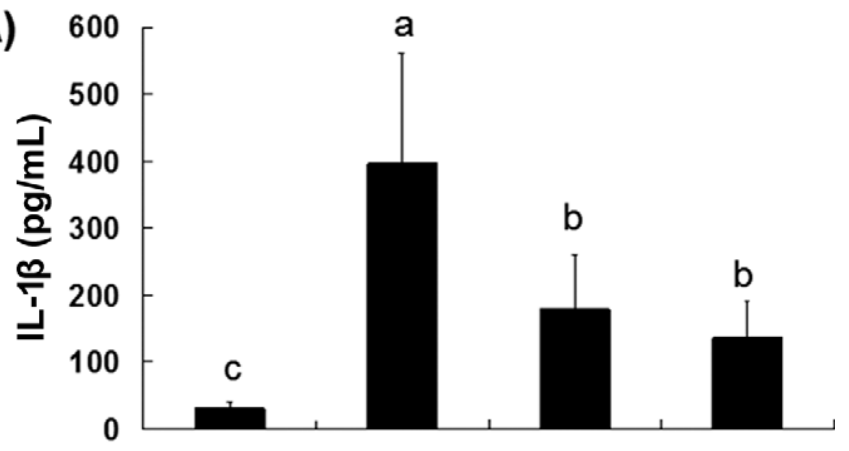

(B)

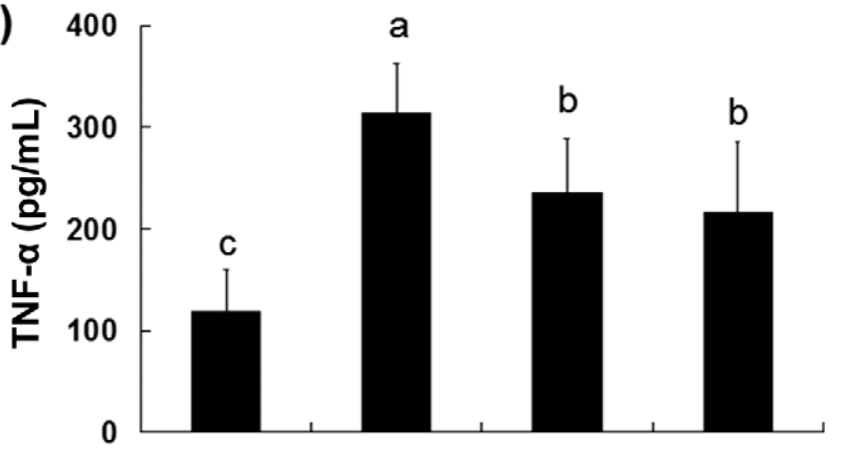

(C)

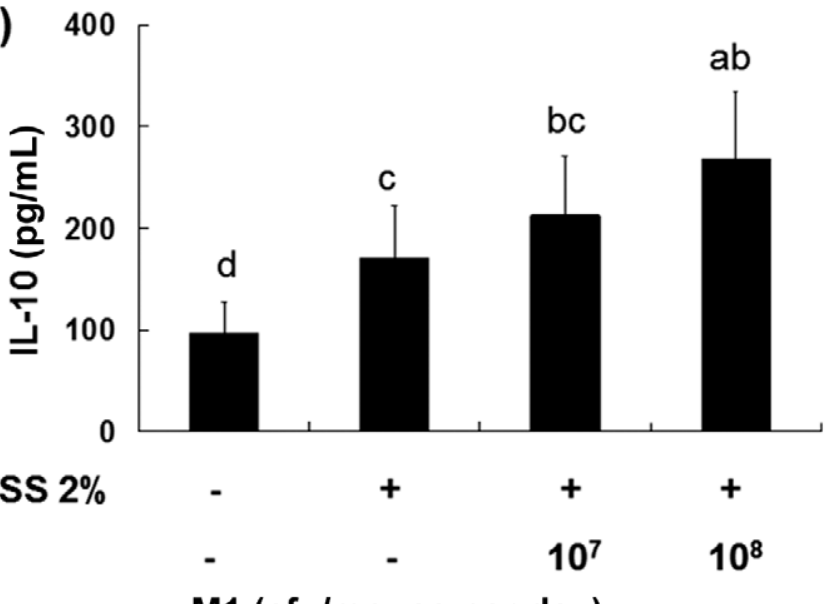

Figure 6. Cytokine analysis of colon organ culture ex vivo. (A) IL$1 \beta$, (B) tumor necrosis factor- $\alpha$ (TNF- $\alpha$ ), and (C) IL-10 production in the supernatant of 24-h colon organ culture of distal colon of mice with different treatments in dextran sodium sulfate (DSS)-induced colitis. Means for data without a common letter differ significantly $(P<0.05)$.

in intestinal epithelial repair, restitution, and migration (Vongsa et al., 2009; Agle et al., 2010) in the Caco-2 monolayer. Both chemokines expressed in the intestine are known to increase the TEER in the intestinal epithelial monolayer (Vongsa et al., 2009). In this study, $L b$. kefiranofaciens M1 significantly upregulated the level of CCL-20 at both the apical and basolateral sites (Figure 2). Conversely, the production of CXCL-12
(A)

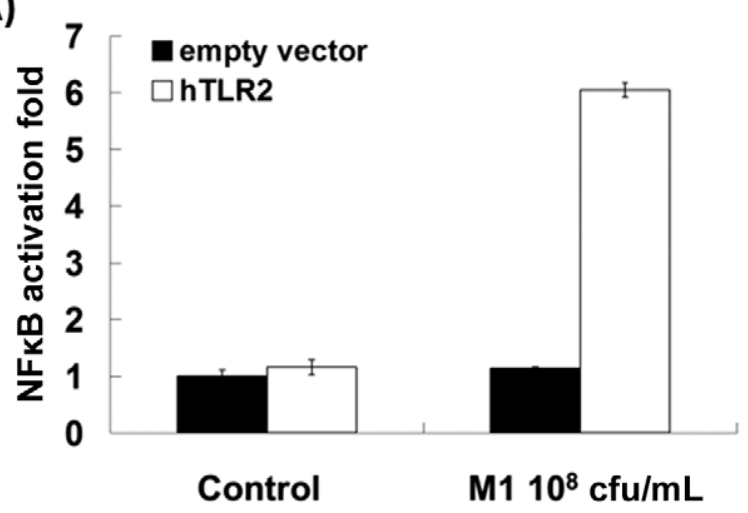

(B)
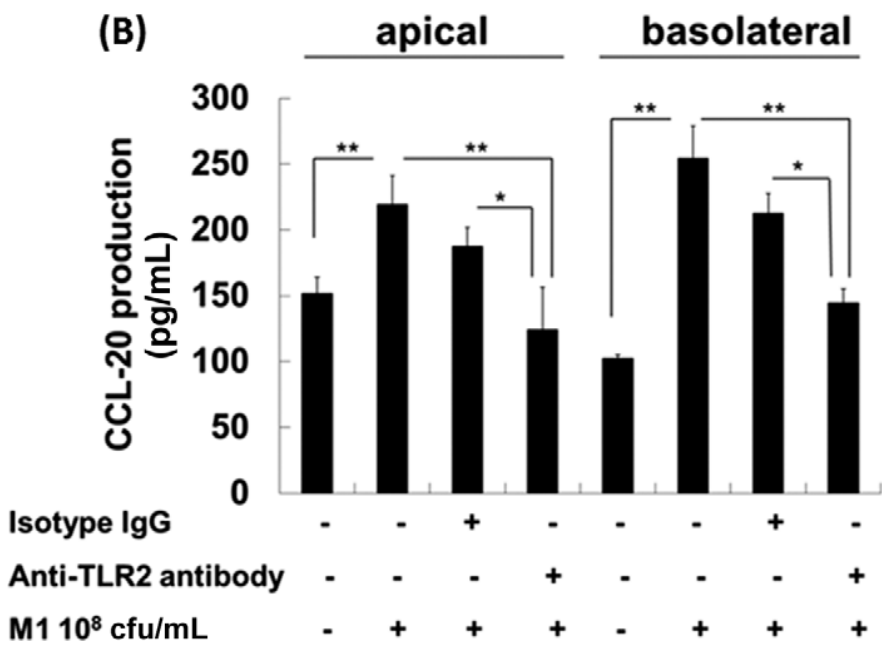

Figure 7. Toll-like receptor (TLR)-2 is a putative receptor for Lactobacillus kefiranofaciens M1. (A) Lb. kefiranofaciens M1 activates nuclear factor $\kappa \mathrm{B}(\mathrm{NF} \kappa \mathrm{B})$ through TLR2 in reporting gene assay; (B) TLR2-specific antibody blocks $L b$. kefiranofaciens M1-induced CCL20 production in Caco-2 monolayer. ${ }^{*} P<0.05 ;{ }^{*} P<0.01$.

showed no change after treatment with $L b$. kefiranofaciens M1. Vongsa et al. (2009) indicated that CCL-20 works through the chemokine receptor CCR6 to activate monomeric RhoGTPase and phosphoinositide 3-kinase, and mobilize intracellular calcium to evoke reorganization of the actin cytoskeleton. Activation of these regulatory pathways contributes to efficient epithelial migration and mucosal barrier repair. Accordingly, increasing CCL-20 production suggests that CCL-20 might be involved in the $L b$. kefiranofaciens M1-mediated enhancement of the TEER. Interleukin-8, a member of the $\mathrm{C}-\mathrm{X}-\mathrm{C}$ chemokine family, is one of the early mediators of inflammatory responses in intestinal epithelial cells. Interleukin-8 causes the over-recruitment and transmigration of neutrophils into inflamed tissue following damage to the epithelium (Struyf et al., 2005). Production of IL-8 did not differ significantly 

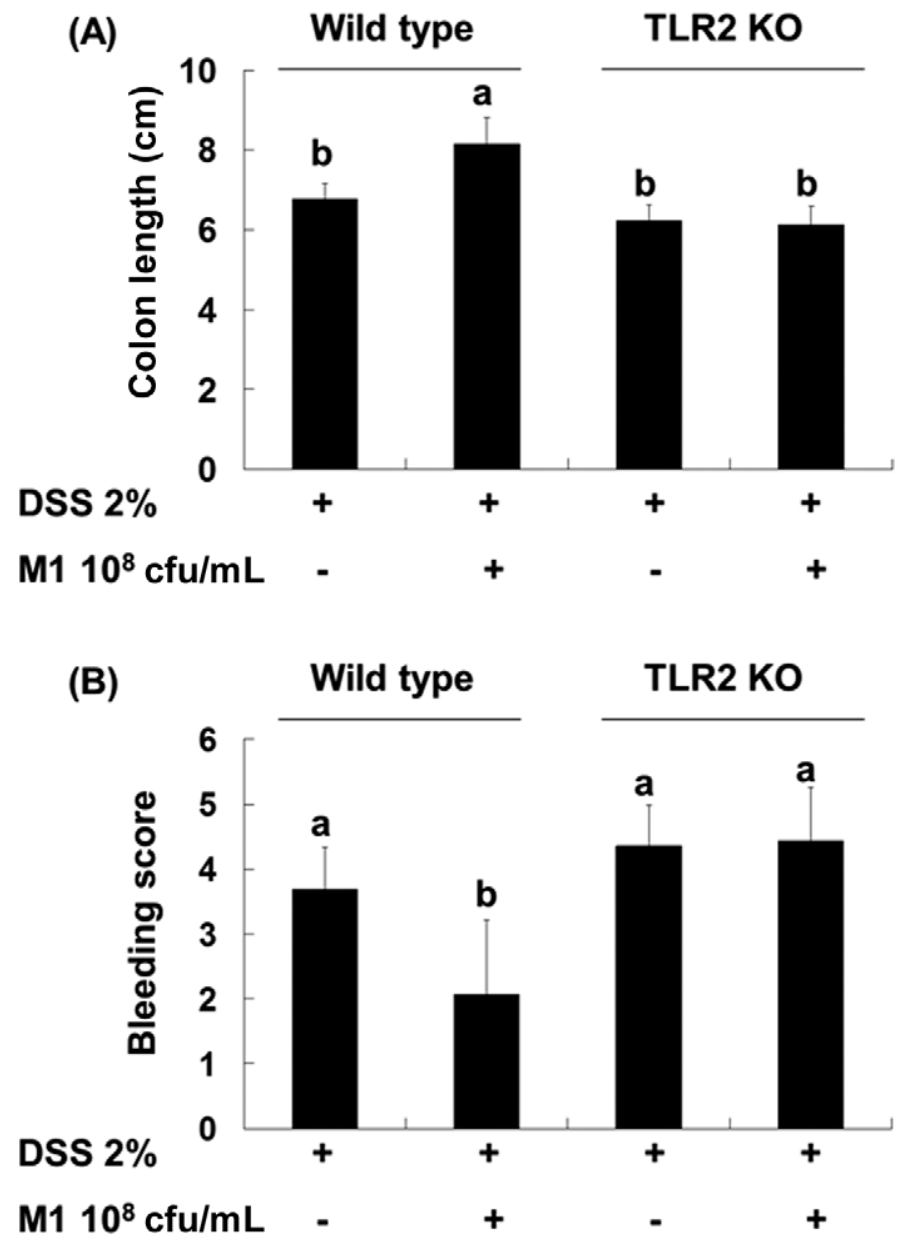

Figure 8. Lactobacillus kefiranofaciens M1 cannot ameliorate dextran sodium sulfate (DSS)-induced colitis in toll-like receptor (TLR)-2 knockout (KO) mice. The bleeding score (B) was evaluated at the last day of treatment. After the mice were killed, the colon of each mouse was removed and the length of colon was measured (A). Means for data without a common letter differ significantly $(P<0.05)$.

among various treatments with $L b$. kefiranofaciens M1 at either the apical or the basolateral sites in the monolayer, which indicated that Lb. kefiranofaciens M1 did not induce an inflammatory status in intestinal epithelial cells (Figure 2).

In the DSS treatment test, our data clearly showed that preincubation of Caco-2 cell monolayers with viable Lb. kefiranofaciens M1 attenuated the decline in TEER induced by DSS (Figure 3). The macromolecule permeability of Caco- 2 cell monolayers was also significantly decreased when cells were exposed to $3 \%$ DSS for 18 h. These findings are supported by several studies using other probiotic bacteria. Pretreatment of T84 cells with Lb. acidophilus R0052 and Lb. rhamnosus R0011 reduced the pathogen-induced decline in TEER (Sherman et al., 2005). Other investigators indicated that the Salmonella enteritidis-induced decrease in TEER was inhibited when Caco-2 monolayers were preincubated with Lactobacillus gasseri (Fajdiga et al., 2006). Koninkx et al. (2010) demonstrated that probiotic bacteria improved the mucosal integrity of enterocyte-like Caco-2 cells after exposure to Salmonella enteritidis. It was also reported that $L b$. plantarum could protect the Caco-2 monolayer from phorbol 12,13-dibutyrate, another barrier-disrupting chemical reagent that induces TEER loss and macromolecular permeability (Karczewski et al., 2010). Our in vitro results verified that Lb. kefiranofaciens M1 has the ability to restore barrier function against DSS-induced damage by increasing the chemokine CCL-20 and reducing the permeability of the intestine in vitro.

The in vivo effects of $L b$. kefiranofaciens M1 on the regulation of intestinal physiology demonstrated that Lb. kefiranofaciens M1 could ameliorate DSS-induced colitis with a significant attenuation in the bleeding score and colon shortening (Figure 4). Histological analysis also showed crypt regeneration and epithelial restitution in the colon in Lb. kefiranofaciens M1treated groups, consistent with $L b$. kefiranofaciens M1 influencing recovery from DSS-induced colitis (Figure $5)$. The spleens of DSS-treated mice were heavier than those of PBS controls. The group pretreated with $10^{8}$ $\mathrm{cfu} / \mathrm{d}$ of Lb. kefiranofaciens M1 showed a spleen weight similar to that of the non-DSS PBS controls, but no significant differences were found among all groups (Figure 4). The spleen is an important organ in immune responses. The increase in spleen weight can be explained by immune activation or infection leading to hyper-activities in this organ (Herías et al., 2005). Other studies have used the DSS-induced colitis model to investigate probiotics with potential in treating colitis. Miyauchi et al. (2009) reported that Lb. rhamnosus could ameliorate DSS-induced colitis through strengthening of the epithelial barrier. Fitzpatrick et al. (2008) found that $E$. coli M-17 could improve DSS-induced colitis and inhibit proinflammatory cytokine produc-

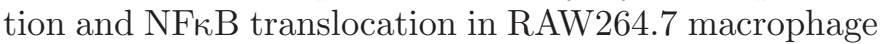
cells treated with LPS.

The acute state of DSS-induced colitis is inclined toward a T-helper 1 response (Strober et al., 2002). Previous studies (Nanda Kumar et al., 2008) have suggested that the presence of certain strains of probiotic bacteria can alter cytokine secretion from a proinflammatory to an antiinflammatory profile in DSS-treated mice. In accordance with these findings, the production of proinflammatory cytokines (TNF- $\alpha$, IL-1 $\beta$ ) decreased and that of the antiinflammatory cytokine (IL-10) increased in the DSS-treated mice given Lb. kefiranofaciens M1 (Figure 6). This evidence suggests that $L b$. kefiranofaciens M1 has a potent direct antiinflammatory activity on epithelial cells, which may lead to inhibition of 
neutrophil accumulation in the mucosal region in DSScolitis mice. Earlier reports have shown that critical proinflammatory mediators such as TNF- $\alpha$ contribute to tissue damage in DSS-induced colitis (Kojouharoff et al., 1997; Obermeier et al., 1999). Interleukin-10 plays a key role in the attenuation of inflammatory responses to intestinal antigens and increases tolerance of $\mathrm{T}$ cells to resident intestinal bacteria. Ulisse et al. (2001) showed an increased tissue level of IL-10 in pouch patients treated with the probiotic VSL\#3 therapy. The therapeutic benefit of probiotic VSL\#3 was also shown by experimental studies in IL-10 knockout mice (Madsen et al., 1999, 2001).

Toll-like receptor 2 is known to recognize many gram-positive bacteria, including lactobacilli, and has recently been shown to elicit protective effects on intestinal epithelial cells in vitro (Cario et al., 2004) and in vivo (Rakoff-Nahoum et al., 2004; Cario et al., 2007). In our study, TLR2 was shown as a putative receptor for Lb. kefiranofaciens M1 in a reporter gene assay (Figure 7A) and was responsible for Lb. kefiranofaciens M1mediated CCL-20 production in the TLR2 antibody blocking assay of the Caco-2 monolayer (Figure 7B). In the animal model, Lb. kefiranofaciens M1 ameliorated DSS-induced colitis in wild-type mice but failed to do so in TLR2 knockout mice (Figure 8). These findings indicate that TLR2 is a functional receptor for the protective ability of Lb. kefiranofaciens M1. Activation of TLR2 may also be a therapeutic method or a selection criterion for potential probiotic strain-bearing intestinal protective function. Furthermore, in the present study, Lactobacillus kefiranofaciens M1 could activate TLR2 (Figure 7A) and induce higher IL-10 production in DSS-induced colitis (Figure 6). A few investigations have indicated that TLR2 is involved in probioticinduced cytokine production in vitro (Cai et al., 2010), especially in regulating antiinflammatory cytokine IL10 production through TLR2-ERK activation (Kaji et al., 2010).

\section{CONCLUSIONS}

In this study, the kefir lactobacillus Lb. kefiranofaciens M1 clearly demonstrated an anticolitis effect. The underlying mechanisms appear to affect intestinal epithelial cells directly, based on the data from the intestinal monolayer in vitro and DSS-induced colitis in vivo. Lactobacillus kefiranofaciens M1 could restore barrier function and reduce the permeability of the intestine against DSS-induced damage by increasing chemokine CCL-20, upregulating the production of antiinflammatory cytokine IL-10, and suppressing the secretion of proinflammatory cytokines through TLR2. To the best of our knowledge, this is the first study to investigate the anticolitis effect of $L b$. kefiranofaciens M1 in vitro, in vivo, and ex vivo. Studies of the detailed underlying mechanisms, such as signaling pathways, are necessary to address the intestinal epithelial protecting effects of this strain.

\section{ACKNOWLEDGMENTS}

We thank the National Science Council in Taiwan for grant support. We thank Shu-Mei Liang in Academia Sinica in Taiwan for providing TLR2 knock-out mice and Ming-Fan Wu in Laboratory Animal Center, National Taiwan University College of Medicine, for his assistance in histological assessment.

\section{REFERENCES}

Agle, K. A., R. A. Vongsa, and M. B. Dwinell. 2010. Calcium mobilization triggered by the chemokine CXCL12 regulates migration in wounded intestinal epithelial monolayers. J. Biol. Chem. 285:16066-16075.

Anderson, R. C., A. L. Cookson, W. C. McNabb, W. J. Kelly, and N. C. Roy. 2010. Lactobacillus plantarum DSM 2648 is a potential probiotic that enhances intestinal barrier function. FEMS Microbiol. Lett. 309:184-192.

Araki, Y., H. Sugihara, and T. Hattori. 2006. In vitro effects of dextran sulfate sodium on a Caco-2 cell line and plausible mechanisms for dextran sulfate sodium-induced colitis. Oncol. Rep. 16:13571362 .

Caballero-Franco, C., K. Keller, S. C. De, and K. Chadee. 2007. The VSL\#3 probiotic formula induces mucin gene expression and secretion in colonic epithelial cells. Am. J. Physiol. Gastrointest. Liver Physiol. 292:G315-G322.

Cai, S., B. H. Bay, Y. K. Lee, J. Lu, and R. Mahendran. 2010. Live and lyophilized Lactobacillus species elicit differential immunomodulatory effects on immune cells. FEMS Microbiol. Lett. 302:189-196.

Cario, E., G. Gerken, and D. K. Podolsky. 2004. Toll-like receptor 2 enhances ZO-1-associated intestinal epithelial barrier integrity via protein kinase C. Gastroenterology 127:224-238.

Cario, E., G. Gerken, and D. K. Podolsky. 2007. Toll-like receptor 2 controls mucosal inflammation by regulating epithelial barrier function. Gastroenterology 132:1359-1374.

Castagliuolo, I., F. Galeazzi, S. Ferrari, M. Elli, P. Brun, A. Cavaggioni, D. Tormen, G. C. Sturniolo, L. Morelli, and G. Palù. 2005. Beneficial effect of auto-aggregating Lactobacillus crispatus on experimentally induced colitis in mice. FEMS Immunol. Med. Microbiol. 43:197-204.

Chen, H. C., S. Y. Wang, and M. J. Chen. 2008a. Microbiological study of lactic acid bacteria in kefir grains by culture-dependent and culture-independent methods. Food Microbiol. 25:492-501.

Chen, L. L., X. H. Wamg, G. H. Lian, J. Zhang, C. H. Ouyang, and F. G. Lu. 2009. Therapeutic effects of four strains of probiotics on experimental colitis in mice. World J. Gastroenterol. 15:321-327.

Fajdiga, S., J. F. J. G. Koninkx, P. C. J. Tooten, and R. MarnšekLogar. 2006. Interference of Salmonella enteritidis and Lactobacillus spp. with IL-8 levels and transepithelial electrical resistance of enterocyte-like Caco-2 cells. Folia Microbiol. (Praha) 51:268-272.

Farnworth, E. R., and I. Mainville. 2003. Kefir: A fermented milk product. Pages 77-111 in Handbook of Fermented Functional Foods. CRC Press, Boca Raton, FL.

Fitzpatrick, L. R., J. Small, R. A. Hoerr, E. F. Bostwick, L. Maines, and W. A. Koltun. 2008. In vitro and in vivo effects of the probiotic Escherichia coli strain M-17: Immunomodulation and attenuation of murine colitis. Br. J. Nutr. 100:530-541.

Gareau, M. G., P. M. Sherman, and W. A. Walker. 2010. Probiotics and the gut microbiota in intestinal health and disease. Nat. Rev. Gastroenterol. Hepatol. 7:503-514. 
Geier, M. S., R. N. Butler, and G. S. Howarth. 2007. Inflammatory bowel disease: Current insights into pathogenesis and new therapeutic options: Probiotics, prebiotics and synbiotics. Int. J. Food Microbiol. 115:1-11.

Gibson, D. L., C. Ma, C. M. Rosenberger, K. S. B. Bergstrom, Y. Valdez, J. T. Huang, M. A. Khan, and B. A. Vallance. 2008. Tolllike receptor 2 plays a critical role in maintaining mucosal integrity during Citrobacter rodentium-induced colitis. Cell. Microbiol. 10:388-403.

Herías, M. V., J. F. J. G. Koninkx, J. G. Vos, J. H. J. Huis in’t Veld, and J. E. van Dijk. 2005. Probiotic effects of Lactobacillus casei on DSS-induced ulcerative colitis in mice. Int. J. Food Microbiol. 103:143-155

Hoarau, C., L. Martin, D. Faugaret, C. Baron, A. Dauba, C. AubertJacquin, F. Velge-Roussel, and Y. Lebranchu. 2008. Supernatant from Bifidobacterium differentially modulates transduction signaling pathways for biological functions of human dendritic cells. PLoS ONE 3:e2753.

Hong, W. S., H. C. Chen, Y. P. Chen, and M. J. Chen. 2009. Effects of kefir supernant and lactic acid bacteria isolated from kefir grain on cytokine production by macrophage. Int. Dairy J. 19:244-251.

Johnson-Henry, K. C., K. A. Donato, G. Shen-Tu, M. Gordanpour, and P. M. Sherman. 2008. Lactobacillus rhamnosus strain GG prevents enterohemorrhagic Escherichia coli O157:H7-induced changes in epithelial barrier function. Infect. Immun. 76:1340-1348.

Kaji, R., J. Kiyoshima-Shibata, M. Nagaoka, M. Nanno, and K. Shida. 2010. Bacterial teichoic acids reserve predominant IL-12 production induced by certain Lactobacillus strains into predominant IL-10 production via TLR2-dependent ERK activation in macrophages. J. Immunol. 184:3505-3513.

Karczewski, J., F. J. Troost, I. Konings, J. Dekker, M. Kleerebezem, R.-J. M. Brummer, and J. M. Wells. 2010. Regulation of human epithelial tight junction proteins by Lactobacillus plantarum in vivo and protective effects on the epithelial barrier. Am. J. Physiol. Gastrointest. Liver Physiol. 298:G851-G859.

Klingberg, T. D., M. H. Pedersen, A. Cencič, and B. B. Budde. 2005. Application of measurements of transepithelial electrical resistance of intestinal epithelial cell monolayers to evaluate probiotic activity. Appl. Environ. Microbiol. 71:7528-7530.

Kojouharoff, G., W. Hans, F. Obermeier, D. N. Männel, T. Andus, J. Schölmerich, V. Gross, and W. Falk. 1997. Neutralization of tumour necrosis factor (TNF) but not of IL-1 reduces inflammation in chronic dextran sulphate sodium-induced colitis in mice. Clin. Exp. Immunol. 107:353-358.

Koninkx, J. F. J. G., P. C. J. Tooten, and G. G. Malago. 2010. Probiotic bacteria induced improvement of the mucosal integrity of enterocyte-like Caco-2 cells after exposure to Salmonella enteritidis 857. J. Funct. Foods 2:225-234.

Li, N., V. G. DeMarco, C. M. West, and J. Neu. 2003. Glutamine supports recovery from loss of transepithelial resitance and increase of permeability induced by media change in Caco-2 cells. J. Nutr. Biochem. 14:401-408.

Liu, J. R., M. J. Chen, and C. W. Lin. 2005. Antimutagenic and antioxidant properties of milk-kefir and soymilk-kefir. J. Agric. Food Chem. 53:2467-2474

Liu, J. R., S. Y. Wang, M. J. Chen, H. L. Chen, P. Y. Yueh, and C. W. Lin. 2006. Hypocholesterolaemic effects of milk-kefir and soyamilkkefir in cholesterol-fed hamsters. Br. J. Nutr. 95:939-946.

Liu, J. R., S. Y. Wang, Y. Y. Lin, and C. W. Lin. 2002. Antitumor activity of milk kefir and soy milk kefir in tumor-bearing mice. Nutr. Cancer 44:183-187.

Madsen, K., A. Cornish, P. Soper, C. McKaigney, H. Jijon, C. Yachimec, J. Doyle, L. Jewell, and C. De Simone. 2001. Probiotic bacteria enhance murine and human intestinal epithelial barrier function. Gastroenterology 121:580-591.

Madsen, K. L., J. S. Doyle, L. D. Jewell, M. M. Tavernini, and R. N. Fedorak. 1999. Lactobacillus species prevents colitis in interleukin 10 gene-deficient mice. Gastroenterology 116:1107-1114.

Marchiando, A. M., W. V. Graham, and J. R. Turner. 2010. Epithelial barriers in homeostasis and disease. Annu. Rev. Pathol. $5: 119-144$.
Meng, G., M. Rutz, M. Schiemann, J. Metzger, A. Grabiec, R. Schwandner, P. B. Luppa, F. Ebel, D. H. Busch, S. Bauer, H. Wagner, and C. J. Kirschning. 2004. Antagonistic antibody prevent toll-like receptor 2-driven lethal shock-like syndromes. J. Clin. Invest. 113:1473-1481.

Mileti, E., G. Matteoli, L. D. Iliev, and M. Rescigno. 2009. Comparison of the immunomodulatory properties of three probiotic strains of lactobacilli using complex culture systems: Prediction for in vivo efficacy. PLoS ONE 4:e7056.

Miyauchi, E., H. Morita, and S. Tanabe. 2009. Lactobacillus rhamnosus alleviates intestinal barrier dysfunction in part by increasing expression of zonula occludens-1 and myosin light-chain kinase in vivo. J. Dairy Sci. 92:2400-2408.

Nanda Kumar, N. S. N., R. Balamurugan, K. Jayakanthan, A. Pulimood, S. Pugazhendhi, and B. S. Ramakrishna. 2008. Probiotic administration alters the gut flora and attenuates colitis in mice administered dextran sodium sulfate. J. Gastroenterol. Hepatol. 23:1834-1839.

Neish, A. S. 2004. Molecular aspects of intestinal epithelial cell-bacterial interactions that determine the development of intestinal inflammation. Inflamm. Bowel Dis. 10:159-168.

Ng, S. C., A. L. Hart, M. A. Kamm, A. J. Stagg, and S. C. Knight. 2009. Mechanisms of action of probiotics: Recent advances. Inflamm. Bowel Dis. 15:300-310.

Obermeier, F., G. Kojouharoff, W. Hans, J. Scholmerich, V. Gross, and W. Falk. 1999. Interferon-gamma (IFN-gamma)- and tumour necrosis factor (TNF)-induced nitric oxide as toxic effector molecule in chronic dextran sulphate sodium (DSS)-induced colitis in mice. Clin. Exp. Immunol. 116:238-245.

Pronio, A., C. Montesani, C. Butteroni, S. Vecchione, G. Mumolo, A. Vestri, D. Vitolo, and M. Boirivant. 2008. Probiotic administration in patients with ileal pouch-anal anastomosis for ulcerative colitis is associated with expansion of mucosal regulatory cells. Inflamm. Bowel Dis. 14:662-668

Rakoff-Nahoum, S., J. Paglino, F. Eslami-Varzaneh, S. Edberg, and R. Medzhitov. 2004. Recognition of commensal microflora by toll-like receptors is required for intestinal homeostasis. Cell 118:229-241.

Reiff, C., M. Delday, G. Rucklidge, M. Reid, G. Duncan, S. Wohlgemuth, G. Hormannsperger, G. Loh, M. Blaut, E. Collie-Duguid, D. Haller, and D. Kelly. 2009. Balancing inflammatory, lipid and xenobiotic signaling pathways by VSL\#3, a biotherapeutic agent, in the treatment of inflammatory bowe disease. Inflamm. Bowel Dis. 15:1721-1736.

Reiff, C., and D. Kelly. 2010. Inflammatory bowel disease, gut bacteria and probiotic therapy. Int. J. Med. Microbiol. 300:25-33.

Resta-Lenert, S., and K. E. Barrett. 2003. Live probiotics protect intestinal epithelial cells from the effects of infection with enteroinvasive Escherichia coli (EIEC). Gut 52:988-997.

Resta-Lenert, S., and K. E. Barrett. 2006. Probiotics and commensals reverse TNF- $\alpha$ - and IFN- $\gamma$-induced dysfunction in human intestinal epithelial cells. Gastroenterology 130:731-746.

Schlee, M., J. Harder, B. Koten, E. F. Stange, J. Wehkamp, and K. Fellermann. 2008. Probiotic lactobacilli and VSL\#3 induce enterocyte beta-defensin 2. Clin. Exp. Immunol. 151:528-535.

Sherman, P. M., K. C. Johnson-Henry, H. P. Yeung, P. S. C. Ngo, J Goulet, and T. A. Tompkins. 2005. Probiotics reduce enterohemorrhagic Escherichia coli O157:H7 and enteropathogenic E. coli O127:H6-induced changes in polarized T84 epithelial cell monolayers by reducing bacterial adhesion and cytoskeletal rearrangements. Infect. Immun. 73:5183-5188.

Strober, W., I. J. Fuss, and R. S. Blumberg. 2002. The immunology of mucosal models of inflammation. Annu. Rev. Immunol. 20:495-549.

Struyf, S., M. Gouwy, C. Dillen, P. Proost, G. Opdenakker, and J. V. Damme. 2005. Chemokines synergize in the recruitment of circulating neutrophils into inflamed tissue. Eur. J. Immunol. 32:1583-1591.

Ukena, S. N., A. Singh, U. Dringenberg, R. Engelhardt, U. Seidler, W. Hansen, A. Bleich, D. Bruder, A. Franzke, G. Rogler, S. Suerbaum, J. Buer, F. Gunzer, and A. M. Westendorf. 2007. Probiotic 
Escherichia coli Nissle 1917 inhibits leaky gut by enhancing mucosal integrity. PLoS ONE 2:e1308.

Ulisse, S., P. Gionchetti, S. D'Alò, F. P. Russo, I. Pesce, G. Ricci, F. Rizzello, U. Helwig, M. G. Cifone, M. Campieri, and C. De Simone. 2001. Expression of cytokines, inducible nitric oxide synthase, and matrix metalloproteinases in pouchitis: Effects of probiotic treatment. Am. J. Gastroenterol. 96:2691-2699.

Vanderpool, C., F. Yan, and B. Polk. 2008. Mechanisms of probiotic action: Implications for therapeutic applications in inflammatory bowel disease. Inflamm. Bowel Dis. 14:1585-1596.

Voltan, S., I. Castagliuolo, M. Elli, S. Longo, P. Brun, R. D’Incà, A. Porzionato, V. Macchi, G. Palù, G. C. Sturniolo, L. Morelli, and D. Martines. 2007. Aggregating phenotype in Lactobacillus crispatus determines intestinal colonization and TLR2 and TLR4 modulation in murine colonic mucosa. Clin. Vaccine Immunol. 14:1138-1148.

Voltan, S., D. Martines, M. Elli, P. Brun, S. Longo, A. Porzionato, V. Macchi, R. D'Incà, M. Scarpa, G. Palù, G. C. Sturniolo, L. Morel- li, and I. Castagliuolo. 2008. Lactobacillus crispatus M247-derived $\mathrm{H}_{2} \mathrm{O}_{2}$ acts as a signal transducing molecule activating peroxisome proliferator activated receptor- $\gamma$ in the intestinal mucosa. Gastroenterology 135:1216-1227.

Vongsa, R. A., N. P. Zimmerman, and M. B. Dwinell. 2009. CCR6 regulation of the actin cytoskeleton orchestrates human beta defensin-2- and CCL20-mediated restitution of colonic epithelial cells. J. Biol. Chem. 284:10034-10045.

Wirtz, S., C. Neufert, B. Weigmann, and M. F. Neurath. 2007. Chemically induced mouse models of intestinal inflammation. Nat. Protoc. $2: 541-546$

Zyrek, A. A., C. Cichon, S. Helms, C. Enders, U. Sonnenborn, and A. Schmidt. 2007. Molecular mechanisms underlying the probiotic effects of Escherichia coli Nissle 1917 involve ZO-2 and PKC $\zeta$ redistribution resulting in tight junction and epithelial barrier repair. Cell. Microbiol. 9:804-816. 